\title{
Modelagem Direta de Integrais de Domínio em Problemas Difusivo-Advectivos usando Funções Radiais no Contexto do Método dos Elementos de Contorno
}

\author{
Loeffler, C. F. ${ }^{*} ;$ Avelar, J. R. ${ }^{2}$ \\ 1 Programa de Pós-Graduação em Engenharia Mecânica, Universidade Federal do Espírito Santo, Vitória, ES, Brasil. \\ 2Vale, Departamento de Locomotivas e Máquinas, Vitória, ES, Brasil. \\ *e-mail: carlosloeffler@bol.com.br
}

\begin{abstract}
Resumo
Este trabalho aplica o Método dos Elementos de Contorno com Interpolação Direta (MECID) onde se interpola diretamente o termo não homogêneo da equação diferencial de governo, para solução de problemas difusivo-advectivos. Empregam-se funções primitivas das funções de interpolação originais no núcleo da integral de domínio, permitindo a transformação desta última numa integral de contorno, semelhantemente ao realizado na técnica de Dupla Reciprocidade, evitando assim a discretização do domínio por meio de células. Esta recente formulação logrou êxito na solução de problemas de grande interesse e sabida dificuldade em engenharia como os de Poisson e de Helmholtz. A aplicabilidade e a precisão do MECID são então testadas na solução no problema de escoamento unidirecional de um fluido sobre um volume controle. Os valores numéricos são comparados com os respectivos valores analíticos.
\end{abstract}

\begin{abstract}
This work is based on the Boundary Element Method with Direct Interpolation (DIBEM) which directly interpolates the inhomogeneous term of the government differential equation, solving diffusive-advective problems. The DIBEM uses a primitive of the original interpolation function in the kernel of the domain integral, allowing the latter processing a boundary integral, similarly to that performed in the Dual Reciprocity Method, thus avoiding domain discretization by cells. The DIBEM has well succeeded in solving well known problems of great interest and difficulty in engineering, such as the governed by the Poisson Equation and the Helmholtz Equation. The applicability and accuracy of the DIBEM are testednow for solving problems characterized by unidirectional fluid flow on a control volume. For this purpose, different meshes are generated to calculate numerically results, whose are compared with the respective analytical solutions.

Keywords: Boundary Element Method, Radial Basis Functions, Direct Interpolation Scheme.
\end{abstract}

\section{Introdução}

O fenômeno da difusão-advecção[1] é muito comum nos problemas de engenharia como, por exemplo: na formação da camada limite de um fluido em escoamentos laminares; na transmissão de calor com a associação entre a propagação choque térmico no meio contínuo e o transporte por meio de fluxo. Estes problemas continuam sendo objeto de atenção e demandam constantes aprimoramento na implementação de métodos numéricos. A aplicação da MECID nestes problemas segue a escala de complexidade tomando-se a Equação de Campo Escalar Generalizada como referência [2].

\section{A Formulação Matemática do MEC}

A aplicação do MEC tem por característica, transformar equações que estão na forma diferencial em equações integrais na forma Integral Inversa [3] e em seguida estabelecer condições em que o problema dependa apenas das condições de contorno para sua solução. No caso em questão, a equação de governo é dada por:

$$
\mathrm{ku}_{, \mathrm{ii}}=\mathrm{v}_{\mathrm{i}} \mathrm{u}_{\mathrm{i}}
$$


O MEC usa como procedimento básico transformar a equação diferencial anterior em equações integrais de contorno. Para isso, em princípio multiplica-se a equação Erro! Fonte de referência não encontrada. por uma função auxiliar $u^{*}(\xi ; X)$, conhecidacomo solução fundamental. Estas funções são assim chamadas por se tratarem da solução analítica de um problema correlato, no qual uma ação concentrada é aplicada num domínio infinito. Assim, $\mathrm{u}^{*}$ seria a solução do seguinte problema:

$$
\mathrm{u}^{*}{ }_{\mathrm{ii}}(\xi ; \mathrm{X})+\mathrm{v}_{\mathrm{i}} \mathrm{u}{ }_{, \mathrm{i}}=-\Delta(\xi ; \mathrm{X})
$$

$\mathrm{Na}$ equação anterior $\Delta$ é a função delta de Dirac, representando uma carga unitária concentrada agindo no ponto denominado ponto fonte e $X$ são as coordenadas de um ponto qualquer denominado ponto campo. Esta função tende ao infinito em qualquer ponto $X=\xi$ e é igual a zero em qualquer outro lugar. Além disso, existem outras propriedades [4].

No entanto, o uso da solução fundamental correlata ao problema difusivo-advectivo proposto, apesar da elegância e precisão (referencia), apresenta problemas por não representar adequadamente problemas em que o campo de velocidades não é constante.

Ao se usar a MECID, a solução fundamental pode ser relacionada a um problema mais simples, correspondendo à solução de um problema correlato ao problema de Laplace. Isto significa uma aproximação no modelo que resulta em relativa perda de precisão. Todavia, os ganhos operacionais costumam compensar a redução na acuidade dos resultados.

Assim, na MECID a abordagem integral referente ao Laplaciano, correspondente ao lado esquerdo da equação Erro! Fonte de referência não encontrada., é similar ao que se faz usualmente aos problemas estritamente difusivos [5], ou seja:

$$
\int_{\Omega} \mathrm{ku}_{{ }_{\mathrm{ii}}} \mathrm{u}^{*} \mathrm{~d} \Omega=\int_{\Gamma} \mathrm{kqu}^{*}(\xi ; \mathrm{X}) \mathrm{d} \Gamma-\int_{\Gamma} \mathrm{kuq}^{*}(\xi ; \mathrm{X}) \mathrm{d} \Gamma-\mathrm{C}(\xi) \mathrm{u}(\xi)(3)
$$

A interpolação da MECID atua de modo específico, particularmente no termo advectivo, cuja forma integral é dada na seguinte forma:

$$
\begin{aligned}
& \int_{\Gamma}\left(v_{\mathrm{i}} u u^{*}\right) \mathrm{n}_{\mathrm{i}} \mathrm{d} \Gamma-\int_{\Omega} \mathrm{v}_{\mathrm{i}} u u_{, \mathrm{i}}^{*} \mathrm{~d} \Omega=\int_{\Gamma}\left(\mathrm{v}_{\mathrm{i}} u \mathrm{u}^{*}\right) \mathrm{n}_{\mathrm{i},} \mathrm{d} \Gamma- \\
& \int_{\Omega}{ }^{\xi} \alpha^{\mathrm{j}} \mathrm{F}^{\mathrm{j}}(\mathrm{X}) \mathrm{d} \Omega=-\int_{\Omega}{ }^{\xi} \alpha^{\mathrm{j}} \Psi_{, \mathrm{ii}}^{\mathrm{j}}(\mathrm{X}) \mathrm{d} \Omega
\end{aligned}
$$

A equação integral completa na forma inversa referente à Equação da Difusão-Advecção é dada por:

$$
\begin{gathered}
-k\left[c(\xi) u(\xi)+\int_{\Gamma}\left(u(X) q^{*}(\xi ; X)-q(X) u^{*}(\xi ; X)\right) d \Gamma\right]= \\
\int_{\Gamma}\left(v_{i} u u^{*}\right) n_{i} d \Gamma-\int_{\Gamma}{ }^{\xi} \alpha^{j} \eta^{J}(\mathrm{X}) \mathrm{d} \Gamma
\end{gathered}
$$

\section{Procedimento de Discretização}

Para obter o sistema de equações do qual os valores de contorno serão calculados numericamente é necessário que a equação (5) seja discretizada. Isto quer dizer que se deve encontrar um número finito de pontos que seja capaz de representar consistentemente odomínio contínuo. Excluindo-se a segunda integral do lado direito da equação (5) o tratamento das integrais segue o procedimento básico do MEC [4]. Já a discretização desta última integral é feita com o auxílio das funções de interpolação, que são funções de base radial, que expressam aproximadamente o comportamento das variáveis do problema entre os pontos eleitos para representar os demais.

As funções de base radial possuem muitas vantagens e por isso seu uso tem crescido enormemente [6]. A simetria radial é das mais importantes propriedades dessa classe de funções; o valor da função aproximante depende tão somente da distância do argumento ao ponto base e quaisquer rotações não influenciam seu resultado. Além disso, a norma euclidiana também se caracteriza por estabelecer valores sempre positivos para seus resultados.

Escrevendo-se toda a equação de governo para um dado ponto fonte $\xi$ genérico, tem-se:

$$
\begin{gathered}
k\left[C(\xi) u(\xi)+H_{\xi 1} u_{1}+H_{\xi 2} u_{2}+\cdots+H_{\xi n} u_{n}-G_{\xi 1} q_{1}-\right. \\
\left.G_{\xi 2} q_{2}-\cdots-G_{\xi n} q_{n}\right]+\left(v_{1} n_{1}+v_{2} n_{2}\right)^{1} G_{\xi 1} u_{1}\left(v_{1} n_{1}+\right. \\
\left.v_{2} n_{2}\right)^{2} G_{\xi 2} u_{2}+\cdots+\left(v_{1} n_{1}+v_{2} n_{2}\right)^{n} G_{\xi n} u_{n}= \\
\xi \alpha^{1} N^{1}+{ }^{\xi} \alpha^{2} N^{2}+\cdots{ }^{\xi} \alpha^{n} N^{n}
\end{gathered}
$$

A discretização resulta na seguinte expressão matricial, já considerando a existência de pontos internos interpolantes, cujas submatrizes estão destacadas naexpressão a seguir:

$$
\begin{gathered}
k\left[\begin{array}{cc}
H_{c c} & 0_{c i} \\
H_{i c} & I_{i i}
\end{array}\right]\left[\begin{array}{l}
u_{c} \\
u_{i}
\end{array}\right]-k\left[\begin{array}{cc}
G_{c c} & 0_{c i} \\
G_{i c} & 0_{i i}
\end{array}\right]\left[\begin{array}{c}
q_{c} \\
q_{i}
\end{array}\right]+\left[\begin{array}{ll}
G_{c c}^{\prime} & 0_{c i} \\
G_{i c}^{\prime} & G_{i i}^{\prime}
\end{array}\right]\left[\begin{array}{l}
u_{c} \\
u_{i}
\end{array}\right]= \\
{\left[\begin{array}{ccc}
{ }^{1} \alpha^{1} & \ldots & { }^{1} \alpha^{n} \\
\vdots & \ldots & \vdots \\
n^{1} & \ldots & n^{n} \alpha^{n}
\end{array}\right]\left[\begin{array}{c}
N_{1} \\
\vdots \\
N_{n}
\end{array}\right]=\left[\begin{array}{c}
A_{1} \\
\vdots \\
A_{n}
\end{array}\right]}
\end{gathered}
$$

Os valores nodais referentes ao potencial estão implicitamente contidos no vetor $\mathrm{A}_{\xi}$, por isso, a etapa seguinte consiste em explicitá-los. Para tanto, primeiramente, verifica-se que para os $\mathrm{n}$ pontos fonte छtem-se:

$$
A_{\xi}=\left(\begin{array}{lll}
N_{1} & N_{2} \ldots & N_{n}
\end{array}\right)\left(\begin{array}{c}
\xi \\
\alpha_{1} \\
\vdots \\
\xi \alpha_{n}
\end{array}\right)
$$

Pode-se demonstrar que [7]: 


$$
\left[{ }^{\xi} \alpha\right]=[F]^{-1}\left[{ }^{\xi} \Lambda\right][F][\alpha]=[F]^{-1}\left[{ }^{\xi} \Lambda\right][u]
$$

A matriz diagonal ${ }^{\xi} \wedge$ que multiplica o potencial é expressa da seguinte forma:

$$
\left[{ }^{\xi} \Lambda\right]=\left[\begin{array}{ccc}
\left(v_{1}(X) u^{*}{ }_{11}+v_{2}(X) u^{*}{ }_{2}\right)^{1} & \cdots & 0 \\
\vdots & \ddots & \vdots \\
0 & \cdots & \left(v_{1}(X) u^{*}{ }_{1}+v_{2}(X) u^{*}, 2\right)^{n}
\end{array}\right](10)
$$

Desse modo, vetor $A_{\xi}$ pode ser escrito explicitamente em termos dos valores do potencial.

$$
\begin{gathered}
{\left[A_{\xi}\right]=\left(\begin{array}{llll}
N_{1} & N_{2} & \cdots & N_{n}
\end{array}\right)\left[\begin{array}{ccc}
F^{11} & \cdots & F^{1 n} \\
\vdots & \ddots & \vdots \\
F^{n 1} & \cdots & F^{n n}
\end{array}\right]^{-1}} \\
{\left[\begin{array}{cccc}
{ }^{\xi} \Lambda_{1} & 0 & \cdots & 0 \\
0 & { }^{\xi} \Lambda_{2} & \cdots & 0 \\
\vdots & \vdots & \ddots & \vdots \\
0 & 0 & \cdots & { }^{\xi} \Lambda_{n}
\end{array}\right]\left[\begin{array}{cc}
\Phi_{c c} & 0 \\
0 & \Phi_{i i}
\end{array}\right]\left(\begin{array}{l}
u_{c} \\
u_{i}
\end{array}\right)}
\end{gathered}
$$

$\mathrm{Na}$ MECID, os pontos campo $X$ e os pontos fonte $\xi$ não podem ser coincidentes, pois resultaria em singularidade no núcleo do lado direito da equação Erro! Fonte de referência não encontrada.5). Isso ocorre porque, ao contrário das formulações clássicas de contorno, não são feitas integrações no sentido de valor principal, eliminando as singularidades. Assim, com o propósito de evitar a singularidade, os pontos campo e os pontos base são posicionados em posições distintas aos pontos $\xi$ sem que isso onere a solução do problema. Por isso, foi inserida uma submatriz $\Phi$ cuja função é de transferir os valores do potencial calculados nos pontos de informação $X$ para as coordenadas dos pontos fonte $\xi$.Ao final, resulta 0 seguinte sistema:

$$
\begin{gathered}
k\left[\begin{array}{cc}
H_{c c} & 0_{c i} \\
H_{i c} & I_{i i}
\end{array}\right]\left[\begin{array}{l}
u_{c} \\
u_{i}
\end{array}\right]-k\left[\begin{array}{ll}
G_{c c} & 0_{c i} \\
G_{i c} & 0_{i i}
\end{array}\right]\left[\begin{array}{l}
q_{c} \\
q_{i}
\end{array}\right]+\left[\begin{array}{ll}
G_{c c}^{\prime} & 0_{c i} \\
G_{i c}^{\prime} & G_{i i}^{\prime}
\end{array}\right]\left[\begin{array}{l}
u_{c} \\
u_{i}
\end{array}\right]= \\
{\left[\begin{array}{ll}
C_{c c} & C_{c i} \\
C_{i c} & C_{i i}
\end{array}\right]\left[\begin{array}{l}
u_{c} \\
u_{i}
\end{array}\right]}
\end{gathered}
$$

\section{Simulação Numérica}

Os testes foram feitos na solução de um problema unidimensional simples, correspondente a um volume de controle quadrado de lado unitário.

Não obstante 0 problema ser fisicamente unidimensional, a malha de elementos de contorno é bidimensional e a imposição de condições de contorno adequadas - Neumann nulo em duas arestas paralelas - garante que o fluxo de calor se direcione numa única direção.

Duas malhas com 32 e 80 elementos de contorno, nas quais diferentes quantidades de pontos internos interpolantes e de pontos fontes internos (pf) foram utilizadas totalizando em 42 malhas utilizadas em ambos os problemas.
Foram colocados num mesmo gráfico de erro médio percentual os valores calculados para a mesma quantidade de pontos no contorno e pontos fontes internos, variando somente a quantidade de pontos interpolantes com a intenção de verificar a influência destes.

Utilizou-se o chamado erro relativo percentual onde se obtém a diferença entre os valores aproximado e exato, dividia pelo maior valor exato. A Figura 1 mostra as características do volume de controle em que feita a simulação numérica com a MECID.

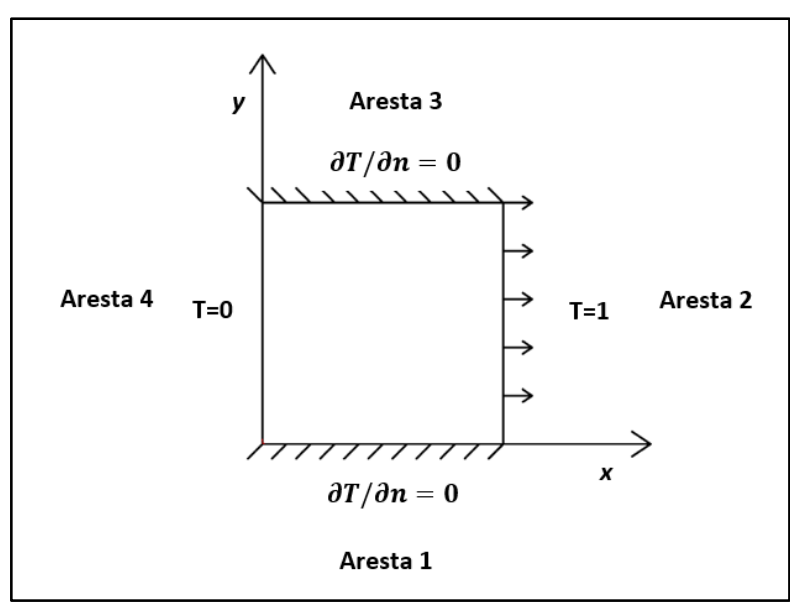

Figura 1-Difusão-advecção unidimensional unidirecional em um volume de controle com lados unitários

Os resultados para o erro médio percentual na aresta 1 , cometido no cálculo do potencial - que representa a temperatura - é mostrado no gráfico da Figura 2.

A convenção usada nesta figura considera as malhas com pontos nodais de contorno e quantidade de pontos fonte no interior (pn/pf) em função da quantidade de pontos interpolantes.

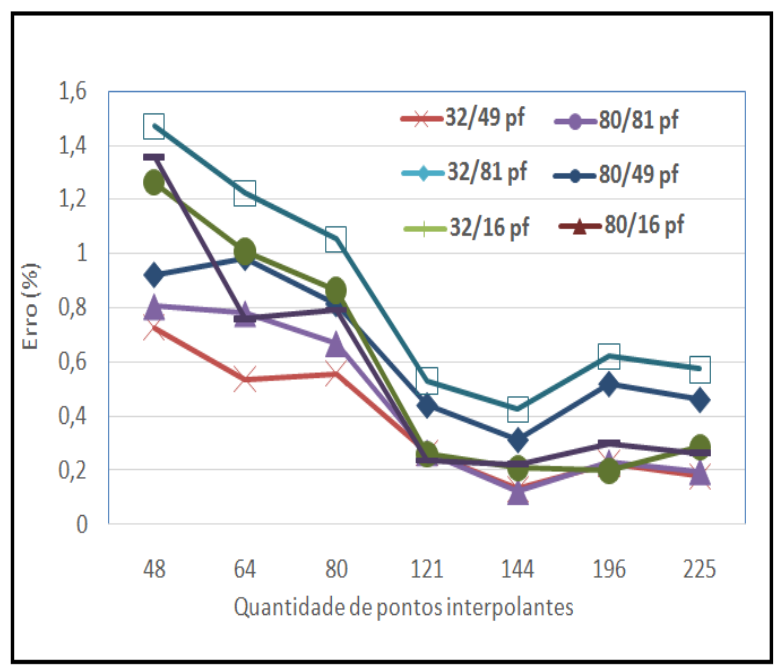

Figura 2 - Gráfico do erro percentual no cálculo do potencial nas arestas horizontais.

Observa-se que os erros no potencial das arestas horizontais se reduzem com a introdução de pontos 
internos interpolantes, mas não de modo monotônico. É certo que o reposicionamento dos pontos interpolantes altera significativamente o desempenho do método. A possibilidade de mau condicionamento matricial é remota, com base em simulações bem sucedidas da MECID em outros problemas físicos. Contudo, a proximidade entre os pontos fonte e de interpolação aqui é um fator relevante. Ressalta-se que a singularidade é do tipo hiperbólica, bem mais crítica do que a singularidade logarítmica que ocorre nos problemas governados pela Equação de Helmholtz [8].

\section{Conclusões}

Em trabalhos anteriores, o MECID se mostrou eficiente na resolução de problemas de Poisson e Helmholtz. Entretanto, para problemas difusivos-advectivosaqui abordados, ficou evidente a necessidade alguns aprimoramentos.

De uma forma geral, os resultados mostraram certa instabilidade com a variação tanto na quantidade quanto no posicionamento dos pontos internos interpolantes. Fato este que tornou os resultados insatisfatórios, pois se esperava um decaimento contínuo do erro ao longo do enriquecimento da malha. Particularmente, a redução do erro era esperada pelo fato de haver uma melhor representatividade do interior do domínio quando se aumenta a quantidade de pontos internos interpolantes. Isto é de grande relevância para o MECID uma vez que se diferencia do MECDR devido à proposta de interpolar todo o núcleo de domínio, incluindo a solução fundamental.

A razão mais provável desse comportamento, bem abaixo do esperado, se deve à integração numérica de uma expressão que possui grau de singularidade superior ao apresentado nas aplicações referentes à Equação de Poisson e Helmholtz. Nesses casos, a integral resolvida pelo procedimento MECID consistia de um núcleo composto de um logaritmo - uma função de ordem zero - cuja integral é imprópria convergente, ou seja, não apresenta singularidade após a integração. No problema abordado, a integral possui nível de singularidade maior, ou seja, há a derivada direcional do potencial, que é de ordem inversa igual a um. É, portanto, uma função cuja integração usando pontos fonte e pontos campo relativamente próximos pode não dar resultados com a precisão esperada. Foi o que provavelmente ocorreu.

Recentemente, em trabalho submetido à apreciação, foi estabelecido um esquema de regularização [9] que elimina a singularidade pela adoção de um esquema similar ao de Haddamard(BRAGA, 2006), que teve ótimo desempenho em problemas de Helmholtz, eliminando a exigência de haver dois conjuntos de pontos para eliminar a singularidade na solução fundamental e retornando resultados ainda melhores do que a modelagem sem o esquema de regularização.

\section{Referências}

[1] INCROPERA, F. P.; DE WITT, D. P. Fundamentos de Transferência de Calor e de Massa.5. ed. Rio de Janeiro: LTC, 2003.

[2] LOEFFLER, C. F. Modelos Mecânicos Derivados da Equação de Campo Escalar Generalizada, Revista Militar de Ciência e Tecnologia, Biblioteca do Exército. vol. 4, n. 1, pp. 24-38, 1992.

[3] BREBBIA, C.A., TELLES, J.C., WROBEL, L.C..Boundary Element Techniques. Springer Verlag, 1984.

[4] BREBBIA, C. A., WALKER, S., Boundary Element Techniques in Engineering, Newnes-Butterworths: London, 1980.

[5] BREBBIA, C.A., DOMINGUEZ, J., Boundary Elements - An Introductory Course. WIT Press, 1992.

[6] BUHMANN, M. D. Radial Basis Function: Theory and implementations. [S.I.]: Cambridge Press, 2003.

[7] LOEFFLER, C. F.; CRUZ, A. L.; BULCÃO, A. Direct Use of Radial Basis Interpolation Functions for Modeling Source Terms With the Boundary Element Method. EABE, v. 50, p. 97 108, 2015.

[8] LOEFFLER, C. F., BARCELOS, H. M., MANSUR, W. J., BULCÃO, A. Solving Helmholtz Problems using Direct Radial Basis Function Interpolation with the Boundary Element Method. Engineering Analysis with Boundary Elements, 2015, Vol. 61, pp. 218-225.

[9] LOEFFLER, C. F.; MANSUR, W. J. A Regularization Scheme Applied to the Direct Interpolation Boundary Element Technique with Radial Basis Functions for Solving Eigenvalue Problem Engineering Analysis with Boundary Elements. Submitted to Engineering Analysis with Boundary Elements, 2016.

[10] BRAGA, C. L. R. Physical-Mathematics Notes (in portuguese). Editora Livraria da Física, São Paulo, 2006. 